\title{
EXERCISE INTOLERANCE IN HEART FAILURE WITH PRESERVED EJECTION FRACTION
}

\author{
Anisha A. Gupte, Ph.D. a; Dale J. Hamilton, M.D., \\ aHouston Methodist Research Institute, bHouston Methodist Hospital, Houston, Texas
}

\begin{abstract}
More than 50\% of Americans with heart failure have preserved ejection fraction (HFpEF). Exercise intolerance is a hallmark of HFpEF, but the pathophysiology is not well understood. Diverse etiologies and incomplete mechanistic understanding have resulted in ineffective management strategies to improve the outcomes of HFpEF. Traditional therapies that have been beneficial in the treatment of heart failure with reduced ejection fraction (HFrEF), neurohormonal blockade in particular, have not been effective in treating HFpEF. In this review, we address underlying mechanisms of HFpEF and present the rationale supporting exercise as a component of comprehensive management.
\end{abstract}

\section{Introduction}

It is estimated that 8.5 million Americans will have heart failure (HF) by $2030,{ }^{1}$ and that more than $50 \%$ of them will have preserved ejection fraction $(\mathrm{pEF}) .^{2}$ Heart failure with preserved ejection fraction (HFpEF) is a result of diastolic dysfunction (DD) that limits ventricular filling and reduces cardiac output. However, the EF in this condition is preserved, e.g., exceeding $45 \%$ to $50 \%$, and the hemodynamics result from extensive fibrosis and myocyte stiffening as can be seen with aging and a variety of comorbidities. ${ }^{3}$ HFpEF occurs predominantly in elderly female patients. Those with hypertension and symptoms of metabolic syndrome, including central obesity and type 2 diabetes, are particularly at risk, and estrogen deficiency is emerging as a contributing factor.

Morbidity and mortality from HFpEF are equivalent to or greater than that of HF with reduced ejection fraction (HFrEF). Nonetheless, established neurohormonal-based therapies used for treating HFrEF have not provided favorable clinical outcomes for HFpEF, and clinical trials have failed to meet their primary end points. ${ }^{4}$ In fact, the lack of successful treatment strategies has made it challenging for clinicians to effectively manage HFpEF. ${ }^{5}$ These management challenges result from limited understanding of the underlying pathological mechanisms of HFpEF and its considerable phenotypic heterogeneity. Furthermore, there is a lack of preclinical models for understanding the mechanisms of this disease and identifying potential treatments. Procurement of fresh human heart tissue for metabolic assessment is challenging. ${ }^{6}$ Therefore, despite improvements in clinical outcomes with HFrEF, morbidity and mortality from HFpEF has remained the same, and the prevalence is rapidly increasing.

Exercise intolerance is a major symptom of HFpEF and is often used as an end point or outcome measure for therapeutic trials. Because exercise intolerance can be elusive at first, a clinical question about a patient's ability to carry on a conversation while climbing a flight of stairs can alert the clinician to an emerging problem. The focus of this review is to highlight the role of exercise intolerance and exertional dyspnea in helping clinicians detect HFpEF and to present a mechanistic rationale for including exercise as a component of therapy.

\section{Pathophysiological Mechanisms}

The mechanisms underlying diastolic HF-including myocardial and extramyocardial alterations - are not fully understood. From a bioenergetics perspective, the myocardium is energy-deprived. ${ }^{7}$ Cardiac-specific alterations occurring in diastolic HF include calcium mishandling resulting from delayed/incomplete sarcoplasmic reticulum calcium release or sequestration and contractile malfunction associated with alterations in titin phosphorylation status. Impairments in myocardial and skeletal muscle energy metabolism, inflammation, and extracellular matrix collagen deposition are leading contenders as pathophysiologic mechanisms. ${ }^{8}$ Together, these mechanisms determine the severity and progression of DD. Thus, it would stand to reason that the ideal therapeutic intervention would target altered myocardial bioenergetics, calcium homeostasis, activity of contractile elements, blunt neurohormonal activation, and fibrosis prevention/regression. There have been several disappointing clinical trials using specific medications that target these pathways, but none have demonstrated success. However, exercise is one intervention that is proving to be surprisingly helpful.

Exercise intolerance presents as an early persistent symptom of HFpEF and is a major determinant of reduced quality of life in these patients. In contrast to HFrEF, the mechanisms of decreased exercise capacity in HFpEF are not well understood. Exercise capacity can be calculated during cardiopulmonary exercise testing as the reduction in peak exercise oxygen consumption $\left(\mathrm{VO}_{2}\right)$ measured by analyses of expired gas. Aerobic exercise requires efficient delivery of oxygen to muscles, which depends on a number of physiological processes including oxygenation of blood in the lungs, cardiac output (CO), the oxygen-carrying capacity of blood, and the capacity of tissue to extract oxygen from the blood. Normal adults are capable of increasing their $\mathrm{VO}_{2}$ more than 6-fold during exercise by increasing cardiac stroke volume, heart rate, and the extraction of oxygen from peripheral blood, which patients with HFpEF are unable to achieve (Table 1). ${ }^{9}$

To understand the mechanism of exercise intolerance, Houstis and Lewis ${ }^{10}$ analyzed the pathophysiology by applying the Fick principle and segregating the individual variables from the Fick equation as follows: (1) deficiency in the peak heart rate (HR), (2) deficiency in the peak stroke volume (SV), (3) deficiency in the 


\begin{tabular}{|l|c|c|}
\hline \multicolumn{2}{|c|}{ Reduced Aerobic Capacity with HFp EF } \\
\hline \multirow{2}{*}{ Parameters } & \multicolumn{2}{|c|}{ Exercise } \\
\cline { 2 - 3 } & Non-Failing Heart & HFp EF \\
\hline Heart Rate & $\uparrow$ & $\mathrm{NC} / \uparrow$ \\
\hline EF \% & $\uparrow$ & $\uparrow$ \\
\hline Stroke Vol & $\uparrow$ & $\downarrow$ \\
\hline Cardiac Output & $\mathrm{NC} /$ & $\downarrow$ \\
\hline $\mathrm{PCWP}$ & $\uparrow$ & $\uparrow$ \\
\hline $\mathrm{VO}_{2 \text { max }}$ & $\uparrow$ & $\downarrow$ \\
\hline${\mathrm{A}-\mathrm{V} \mathrm{O}_{2}}_{2}$ gradient & $\uparrow$ & $\downarrow$ \\
\hline
\end{tabular}

Table 1. Exercise parameter changes in subject with non-failing heart versus heart failure with preserved ejection fraction. Although both groups have preserved ejection fraction, impaired oxygen delivery and extraction limits exercise capacity in the latter group. EF\%: systolic ejection fraction; PCWP: pulmonary capillary wedge pressure; $\mathrm{VO}_{2}$ max: maximum oxygen uptake in $\mathrm{mL} / \mathrm{kg} / \mathrm{min}$; A-VO2 gradient: difference between arterial and venous blood oxygen concentration.

peak arteriovenous oxygen difference $\left(\mathrm{AVO}_{2}=\right.$ difference between the arterial and venous oxygen contents), and (4) factors that independently lead to exercise cessation before the Fick variable can reach full reserve potential, for example, inadequate effort and musculoskeletal complaints. This results in the following Fick equation: $\mathrm{VO}_{2}=\mathrm{CO} \times\left(\mathrm{AVO}_{2}\right)=(\mathrm{HR} \times \mathrm{SV}) \times\left(\mathrm{AVO}_{2}\right)$

In HFpEF, several cardiac and noncardiac mechanisms for reduced aerobic capacity have been speculated. Santos et al. ${ }^{11}$ and Abudiab et al. ${ }^{12}$ suggested that the major contributor to exercise inability in HFpEF patients is the reduced cardiac output relative to metabolic needs, indicating a cardiac cause. Extensive fibrosis and titin hypophosphorylation ${ }^{7}$ is thought to create stiffness in the $\mathrm{LV}$ of patients with HFpEF, contributing to the cardiac limitations in exercise capacity. Increased diastolic stiffness prevents the increase in LV end-diastolic volume that normally accompanies exercise, thus patients with HFpEF have a limited ability to use the Frank-Starling mechanism despite greater filling pressures. ${ }^{13}$ Augmentation of relaxation velocity fails to occur as heart rate increases during exercise. Therefore, during exercise, diastolic pressure increases, stroke volume fails to increase, and patients with HFpEF experience dyspnea and fatigue., ${ }^{9,14}$

In contrast, Haykowsly et al. ${ }^{15}$ found that while reduced $\mathrm{VO}_{2}$ at peak exercise was associated with reduced cardiac output, the strongest independent predictor of peak $\mathrm{VO}_{2}$ was the change in $\mathrm{AVO}_{2}$ difference from rest to peak exercise in $\mathrm{HFpEF}$ patients compared to age-matched healthy controls performing exhaustive upright cycling exercise. The impact of $\mathrm{AVO}_{2}$ difference suggests that peripheral, noncardiac factors are important contributors to exercise intolerance in HFpEF patients (Figure 1). In support, Dhakal et al. showed that peripheral oxygen extraction was lower in HFpEF compared to controls and HFrEF patients and was the major determinant of reduced exercise capacity. ${ }^{16}$ The discrepancies in the above studies can be explained by Little and Borlaug, ${ }^{9}$ who observed that although peripheral abnormalities were largely associated with exercise limitations, a cardiac limitation was present and could be the first and foremost underlying cause. It is also likely that common comorbidities associated with HFpEF such as pulmonary disease, obesity, and diabetes may contribute to exercise intolerance independent of the heart. Finally, a common technical problem of submaximal effort due to noncardiac issues can contribute to lower $\mathrm{AVO}_{2}$.

In the early 1980s, complete bed rest and avoidance of exercise was thought to be crucial for HF patients. However, this thought has been challenged in recent years, and supervised exercise is now a recommended therapy, especially for patients with HFpEF. Indeed, contrary to the observed exercise intolerance in HFpEF patients, exercise training has been shown to be an effective therapy in the recent Exercise training in Diastolic Heart Failure (Ex-DHF) trial. ${ }^{17}$ In this study, 64 patients with HFpEF were randomized to receive supervised exercise in addition to the usual care, while 20 received only the usual care for 3 months. Endurance and resistance exercise resulted in improved $\mathrm{VO}_{2}$ max and diastolic function (reduced $\mathrm{E} / \mathrm{e}^{\prime}$ ratios) and atrial reverse remodeling, with an overall improvement in quality of life scores. The benefits of exercise were not due to changes in blood pressure or body mass index since both parameters remained unchanged in both groups for the duration of the study. Other smaller studies and meta-analyses have also reiterated the benefits of exercise training in HFpEF. ${ }^{18,19}$

Even with these improvements, the mechanisms underlying the benefits of exercise in patients with HFpEF are still unclear. One speculation is the improvement of myocardial bioenergetics and metabolism with exercise (Figure 2). The process of myocardial relaxation is an energy-consuming process, highly dependent on the ratio of ADP / ATP and levels of ADP and Pi, all of which should be low enough to facilitate ADP to ATP conversion. Production of ATP is dependent on the ability of efficient substrate oxidation. Diastolic relaxation consumes energy since ATP hydrolysis is required for myosin detachment from actin filaments, $\mathrm{Ca}^{2+}$ dissociation from troponin $\mathrm{C}$, and sarcoplasmic reticulum $\mathrm{Ca}^{2+}$

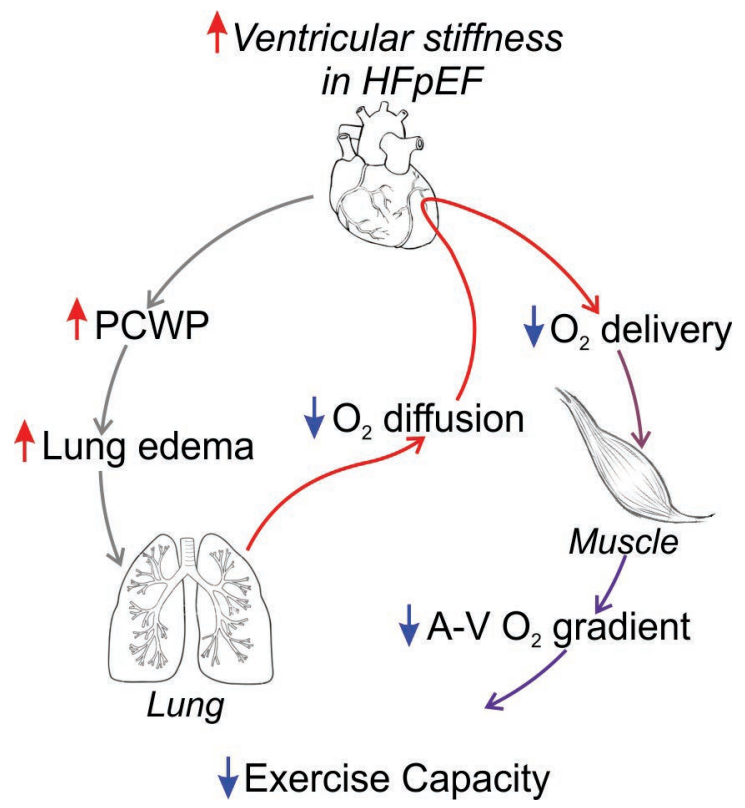

Figure 1. Mechanism for reduced oxygen uptake and delivery in HFpEF. Ventricular stiffness leads to left ventricular increased diastolic filling pressure and PCWP. This results in increased lung water and alveolar edema that limits oxygen diffusion. In turn, there is decreased oxygen delivery due to the combined effects of reduced cardiac output and arterial oxygen content. Peripheral mechanisms lead to impaired extraction of oxygen in skeletal mucscle beds, resulting in decreased exercise capacity. (Figure design by A. Zhang.) HFpEF: heart failure with preserved ejection fraction; PCWP: pulmonary capillary wedge pressure. 


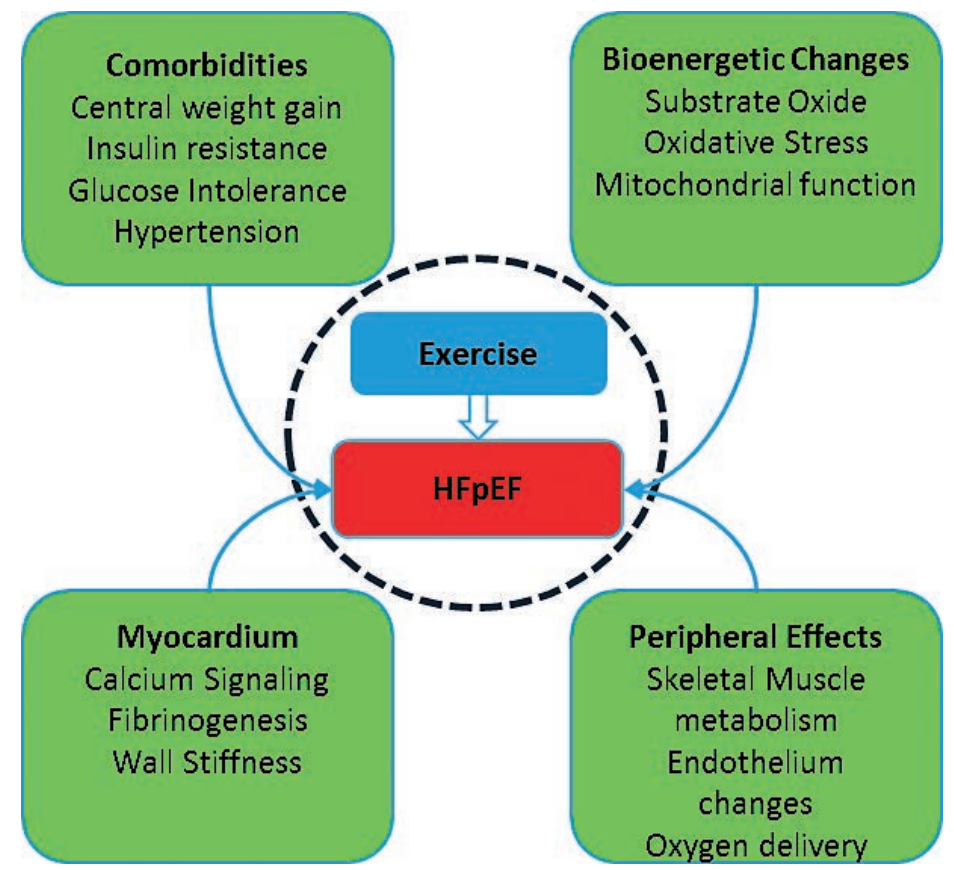

Figure 2. This figure shows comorbidities and bioenergetic, myocardial, peripheral pathogenic processes in HFpEF. Exercise has the potential to reverse pathogenic processes in HFpEF by alleviating these processes and comorbidities. HFpEF: heart failure with preserved ejection fraction.

reuptake. ${ }^{20}$ Thus, diastolic function also depends upon optimal metabolic processes that generate ATP. The commonly associated comorbidities with DD, which include diabetes, hypertrophy secondary to hypertension, and obesity, are all associated with perturbed cardiac metabolism that can reduce the ATP supply required by the relaxing $L V$.

\section{Metabolic Adaptation versus Maladaptation}

The myocardium in diastolic HF undergoes major metabolic changes, but not all have been well characterized. Cardiac positron emission tomography (PET) imaging studies have revealed an increase in fatty acid uptake and a decrease in glucose uptake oxidation with increasing severity of DD. ${ }^{21-23}$ This is different from the greater glucose oxidation seen in HFrEF. However, the excess fatty acid oxidation in HFpEF may lead to overburdening of the mitochondria, accumulation of acylcarnitines, and greater oxidative stress. ${ }^{24}$ Thus, although fatty acid oxidation is increased, there is not a concomitant increase in ATP generation, thereby creating a net energy deficit. Further, this change is replacing the energy-efficient glucose substrate utilization, which can provide a greater number of ATP molecules per molecule of oxygen consumed..$^{25}$ With reduced glucose utilization and incomplete fatty acid oxidation, the myocardium remains energy deprived, and this may underlie the exercise intolerance associated with HFpEF. Enhanced fatty acid uptake and oxidation is followed by mitochondrial uncoupling and production of reactive oxygen species, primarily at the levels of complexes I and III of the mitochondrial respiratory chain. ${ }^{26}$ Oxidative stress can trigger fibrosis processes that result in stiffer ventricles and diminished ventricular relaxation.

In a study by Basu et al., type 1 diabetic Akita mice exhibited DD with preserved systolic function associated with increased myocardial fatty acid oxidation rates and greater accumulation of intramyocardial ceramide and diacylglycerol. ${ }^{27}$ In addition, insulin treatment reversed these metabolic derangements and alleviated DD. ${ }^{27}$ Given that inhibition of fatty acid oxidation appeared to be a promising therapeutic target, several drugs were investigated. For example, perhexiline, an inhibitor of fatty acid oxidation, was shown to correct energy deficiency, $\mathrm{DD}$, and exercise intolerance in patients with hypertrophic cardiomyopathy and dilated cardiomyopathy. ${ }^{28,29}$ However, an emerging view is challenging these treatments by suggesting that reduced glucose uptake and increased fatty acid oxidation is a required metabolic adaptation in the failing heart, and fatty acid oxidation per se should not be blocked. ${ }^{30}$ While increased fatty acid oxidation may be adaptive, the incomplete oxidation and resulting intermediates may be maladaptive. Therefore, it is likely more important to improve the bioenergetics mechanism that enables complete and efficient fatty acid oxidation than to reduce the overall amount of fatty acid oxidation or increase excessive glucose oxidation. In fact, Koves and colleagues have shown that exercise can improve the efficiency of the mitochondrial machinery in complete fatty acid oxidation that results in efficient energy generation. ${ }^{24}$

\section{The Role of Estrogen}

In our laboratory, we have identified a mouse model of diastolic $\mathrm{HF}$ that stems from pressure overload in estrogen-deficient female mice. Ovariectomized (OVX) female C57Bl6 mice, when treated with L-NAME and angiotensin II, get more severe diastolic HFpEF than their counterparts receiving sham surgery and the same treatments. On the other hand, male mice receiving the same treatments get systolic HF with suppressed ejection fraction. Our preliminary unpublished results show that OVX mice with more severe DD have limited mitochondrial function, greater production of mitochondrial reactive oxygen species, and more morphological aberrations in the myocardium, all of which suggest mitochondrial dysfunction. This model represents the growing problem of HFpEF in elderly, postmenopausal women and can be used to investigate the underlying mechanisms. While we have not yet tested the effects of exercise on these mice, we predict that exercise will reverse the bioenergetics limitations and improve diastolic function.

Exercise has been shown to reverse many metabolic derangements in skeletal muscle, leading to more efficient substrate utilization and improvement of systemic outcomes such as insulin resistance and cardiometabolic fitness. ${ }^{31}$ It is likely that exercise benefits in diastolic HF stem from improved bioenergetics in the heart that facilitates efficient fatty acid utilization without the accumulation of acylcarnitines, thereby suppressing mitochondrial oxidative stress and triggers for fibrinogenesis. Exercise may also improve calcium and insulin signaling systemically, which may remove the associated comorbidities that stress the failing heart. As shown in Figure 1, exercise can reverse or counter several pathogenic factors related to HFpEF.

\section{Conclusion}

While exercise intolerance is a prominent feature of DD, supervised exercise may be beneficial in reversing some of its systemic, structural, and metabolic cardiac derangements (Table 2). Further studies are required to determine which kind of exercise would give the maximum benefits and can be reasonably performed by such patients. Further studies are needed to investigate the mechanisms underlying exercise benefits in patients with HFpEF. 
Benefits of Exercise for Individuals with HFpEF

Improved myocardial substrate delivery and oxidation

Improved energetics (ATP/PCr high energy transfer)

Improved comorbidity control (Obesity, HTN, MS/DM, lipids)

Improved peripheral tissue oxygen delivery

Improved exercise capacity

Table 2. Benefits of exercise for individuals with HFpEF. HFpEF: heart failure with preserved ejection fraction; ATP: adenosine triphosphate; PCr: phosphocreatine; HTN: arterial hypertension; MS/DM: metabolic syndrome/ diabetes.

\section{Key Points:}

- HFpEF is diagnostically and therapeutically challenging.

- Pathophysiological mechanisms of HFpEF remain poorly understood.

- Innovative preclinical and translational research methodologies are needed.

- Exercise limitation serves as both an early symptom and therapeutic end point.

- Exercise is a promising therapy to reverse metabolic derangements in HFpEF.

Conflict of Interest Disclosure: The authors have received grant and foundation funding from the American Heart Association, Pat Studdert, Charif Souki, the Stedman-West Foundation, and Elaine and Marvy Finger.

Keywords: heart failure with preserved ejection fraction, impaired diastolic function, exercise intolerance, metabolic and bioenergetic factors, exercise therapy, diastolic dysfunction

\section{References}

1. Heidenreich PA, Albert NM, Allen LA, et al.; American Heart Association Advocacy Coordinating Committee; Council on Arteriosclerosis, Thrombosis and Vascular Biology; Council on Cardiovascular Radiology and Intervention; Council on Clinical Cardiology; Council on Epidemiology and Prevention; Stroke Council. Forecasting the impact of heart failure in the United States: a policy statement from the American Heart Association. Circ Heart Fail. 2013 May;6(3):606-19.

2. Owan TE, Hodge DO, Herges RM, Jacobsen SJ, Roger VL, Redfield MM. Trends in prevalence and outcome of heart failure with preserved ejection fraction. N Engl J Med. $2006 \mathrm{Jul}$ 20;355(3):251-9.

3. Loffredo FS, Nikolova AP, Pancoast JR, Lee RT. Heart failure with preserved ejection fraction: molecular pathways of the aging myocardium. Circ Res. 2014 Jun 20;115(1):97-107.

4. Upadhya B, Taffet GE, Cheng CP, Kitzman DW. Heart failure with preserved ejection fraction in the elderly: scope of the problem. $J$ Mol Cell Cardiol. 2015 Jun;83:73-87.

5. Nanayakkara S, Kaye DM. Management of heart failure with preserved ejection fraction: a review. Clin Ther. 2015 Oct 1;37(10):2186-98.

6. Sharma K, Kass DA. Heart failure with preserved ejection fraction: mechanisms, clinical features, and therapies. Circ Res. 2014 Jun 20;115(1):79-96.

7. Phan TT, Abozguia K, Nallur Shivu G, et al. Heart failure with preserved ejection fraction is characterized by dynamic impairment of active relaxation and contraction of the left ventricle on exercise and associated with myocardial energy deficiency. J Am Coll Cardiol. 2009 Jul 28;54(5):402-9.
8. Chung CS, Hutchinson KR, Methawasin M, et al. Shortening of the elastic tandem immunoglobulin segment of titin leads to diastolic dysfunction. Circulation. 2013 Jul 2;128(1):19-28.

9. Little WC, Borlaug BA. Exercise intolerance in heart failure with preserved ejection fraction: what does the heart have to do with it? Circ Heart Fail. 2015 Mar;8(2):233-5.

10. Houstis NE, Lewis GD. Causes of exercise intolerance in heart failure with preserved ejection fraction: searching for consensus. J Card Fail. 2014 Oct;20(10):762-78.

11. Santos M, Opotowsky AR, Shah AM, Tracy J, Waxman AB, Systrom DM. Central cardiac limit to aerobic capacity in patients with exertional pulmonary venous hypertension: implications for heart failure with preserved ejection fraction. Circ Heart Fail. 2015 Mar;8(2):278-85.

12. Abudiab MM, Redfield MM, Melenovsky V, et al. Cardiac output response to exercise in relation to metabolic demand in heart failure with preserved ejection fraction. Eur $\mathrm{J}$ Heart Fail. 2013 Jul;15(7):776-85.

13. Little WC, Kitzman DW, Cheng CP. Diastolic dysfunction as a cause of exercise intolerance. Heart Fail Rev. 2000 Dec;5(4):301-6.

14. Zile MR, Brutsaert DL. New concepts in diastolic dysfunction and diastolic heart failure: Part I: diagnosis, prognosis, and measurements of diastolic function. Circulation. 2002 Mar 19;105(11):1387-93.

15. Haykowsky MJ, Brubaker PH, John JM, Stewart KP, Morgan TM, Kitzman DW. Determinants of exercise intolerance in elderly heart failure patients with preserved ejection fraction. J Am Coll Cardiol. 2011 Jul 12;58(3):265-74.

16. Dhakal BP, Malhotra R, Murphy RM, et al. Mechanisms of exercise intolerance in heart failure with preserved ejection fraction: the role of abnormal peripheral oxygen extraction. Circ Heart Fail. 2015 Mar;8(2):286-94.

17. Edelmann F, Gelbrich G, Düngen HD, et al. Exercise training improves exercise capacity and diastolic function in patients with heart failure with preserved ejection fraction: results of the Ex-DHF (Exercise training in Diastolic Heart Failure) pilot study. J Am Coll Cardiol. 2011 Oct 18;58(17):1780-91.

18. van Tol BA, Huijsmans RJ, Kroon DW, Schothorst M, Kwakkel G. Effects of exercise training on cardiac performance, exercise capacity and quality of life in patients with heart failure: a metaanalysis. Eur J Heart Fail. 2006 Dec;8(8):841-50.

19. Malfatto G, Branzi G, Osculati G, et al. Improvement in left ventricular diastolic stiffness induced by physical training in patients with dilated cardiomyopathy. J Card Fail. 2009 May; 15(4):327-33.

20. Ussher JR, Jaswal JS. Inhibition of fatty acid oxidation as an approach to treat diastolic heart failure. Heart Metab. 2013;61:20-4.

21. Hamirani YS, Kundu BK, Zhong $M$, et al. Noninvasive Detection of Early Metabolic Left Ventricular Remodeling in Systemic Hypertension. Cardiology. 2016;133(3):157-62.

22. Gupte AA, Hamilton DJ. Molecular Imaging and Precision Medicine. Cardiology. 2016;133(3):178-80.

23. Rijzewijk LJ, van der Meer RW, Lamb HJ, et al. Altered myocardial substrate metabolism and decreased diastolic function in nonischemic human diabetic cardiomyopathy: studies with cardiac positron emission tomography and magnetic resonance imaging. J Am Coll Cardiol. 2009 Oct 13;54(16):1524-32.

24. Koves TR, Ussher JR, Noland RC, et al. Mitochondrial overload and incomplete fatty acid oxidation contribute to skeletal muscle insulin resistance. Cell Metab. 2008 Jan;7(1):45-56. 
25. Barger PM, Kelly DP. Fatty acid utilization in the hypertrophied and failing heart: molecular regulatory mechanisms. Am J Med Sci. 1999 Jul;318(1):36-42.

26. Boudina S, Sena S, Theobald $H$, et al. Mitochondrial energetics in the heart in obesity-related diabetes: direct evidence for increased uncoupled respiration and activation of uncoupling proteins. Diabetes. 2007 Oct;56(10):2457-66.

27. Basu R, Oudit GY, Wang X, et al. Type 1 diabetic cardiomyopathy in the Akita (Ins2WT/C96Y) mouse model is characterized by lipotoxicity and diastolic dysfunction with preserved systolic function. Am J Physiol Heart Circ Physiol. 2009 Dec;297(6):H2096-108.

28. Abozguia K, Elliott P, McKenna W, et al. Metabolic modulator perhexiline corrects energy deficiency and improves exercise capacity in symptomatic hypertrophic cardiomyopathy. Circulation. 2010 Oct 19;122(16):1562-9.

29. Beadle RM, Williams LK, Kuehl M, et al. Improvement in cardiac energetics by perhexiline in heart failure due to dilated cardiomyopathy. JACC Heart Fail. 2015 Mar;3(3): 202-11.

30. Taegtmeyer H, Beauloye C, Harmancey R, Hue L. Insulin resistance protects the heart from fuel overload in dysregulated metabolic states. Am J Physiol Heart Circ Physiol. 2013 Dec;305(12):H1693-7.

31. Huffman KM, Koves TR, Hubal MJ, et al. Metabolite signatures of exercise training in human skeletal muscle relate to mitochondrial remodelling and cardiometabolic fitness. Diabetologia. 2014 Nov;57(11):2282-95. 